\title{
What brings adolescents to the Obstetrics and Gynaecology Emergency Department of a General Hospital
}

\author{
M. Litos, V. Triantafyllidi, D. Vasileiadou, E. Papamargaritis, C. Sofoudis, K. Bakalianou
}

Department of Obstetrics and Gynecology, Agia Olga General Hospital, Athens, Greece

Corresponding Author

Varvara Triantafyllidi, Department of Obstetrics and Gynecology, Konstandopoulio General Hospital, Athens, Greece, v.triantafyllidi@hotmail.com

\section{Abstract}

Introduction: Paediatric \& Adolescent Gynaecology is a special field and also part of the everyday practice. This paper studies the problems adolescent females present with at the Obstetrics \& Gynaecology (O\&G) Emergency Department (ED). Material and Methods: Data regarding adolescent females that were examined at the O\&G ED over one year (2018) were extracted from the records of Konstantiopouleio General Hospital, Athens, Greece. Results: Of the 1534 women that attended O\&G ED over one year, 111 were under 19 years old (7\%). Of the 83 gynaecological cases, the presenting symptom was pelvic pain in $54(65 \%)$, irregular vaginal bleeding in $11(13 \%)$, urinary tract symptoms in $11(13 \%)$, vaginal discomfort in $3(4 \%)$ and others in 4 (5\%). In 39 (47\%) no urgent gynaecological pathology was detected. There were 11 cases $(13 \%)$ of ovarian cysts, 10 of urinary tract infection (12\%), 7 (8\%) of other surgical pathology, 6 (8\%) of first trimester pregnancy complications and 10 others. Of the 28 pregnant adolescents, 10 complained of abdominal pain (36\%), 6 of hyperemesis (21\%), 5 of myometrial activity (18\%), 4 of vaginal bleeding (14\%). In 18 cases (64\%) no acute medical condition was diagnosed, 4 were admitted in active labour (14\%) and one with threatened delivery, two were referred to other specialties and three refused the examination. Conclusion: O\&G emergency conditions in adolescent females are common and potentially serious. Healthcare professionals need to be aware and alert to the special needs of this significant and sensitive population group.

Key words: Puberty, adolescent females, emergency department, obstetrics \& gynaecology

\section{Introduction}

Adolescent females, defined by WHO as 10-19 years old ${ }^{1}$, have special gynaecological problems that constitute the well-established special field of Paediatric and Adolescent Gynaecology. They also 
have usual gynaecological and obstetrical conditions that get managed by general Obstetricians \& Gynaecologists, especially in the emergency setting. The many physical and psychological changes of puberty can have significant impact on the health status of these young women and the provision of health care. Therefore, it is important for the healthcare professionals and services to acknowledge their needs and be prepared to address them. However, due to its otherwise low morbidity, this considerable population group stays out of the spotlight ${ }^{2,3,4}$.

This study aims to investigate the Obstetrical and Gynaecological problems that bring adolescent females to the Emergency Department of a General Hospital.

\section{Materials-Methods}

We studied adolescent patients $(<19$ years old) that presented in the Obstetrics and Gynaecology (O\&G) Emergency Department (ED) of Konstantopouleio General Hospital for a time period of one year (2018).The hospital covers the North West greater metropolitan area of Athens, the capital of Greece, it is medium sized with just under 300 beds and accepts emergency cases on a one in four rota.
Data on symptoms, diagnosis and management of the cases was extracted from the hospital records; it was collected retrospectively and was recorded anonymously.

\section{Results:}

Out of 1534 women that were examined in the $0 \& G$ ED of the hospital during that year, 111 (7\%) were adolescents. Of those 83 (75\%) had gynecological condition and 28 (25\%) were pregnant.

Regarding gynaecological cases, the presenting symptom was abdominal pain in 54 cases $(65 \%)$, menstrual disorders in 11 (13\%), urinary symptoms in 11 (13\%), vaginal discomfort in 3 (4\%) and others in 4 cases (5\%) (Figure 1). In 39 cases (47\%) no gynaecological pathology was diagnosed, 11 were diagnosed with ovarian cysts, intact in $6(7 \%)$ and ruptured in 5 (6\%), 10 cases had urinary tract infection $(12 \%)$ and $7(8 \%)$ had surgical pathology. Viable $1^{\text {st }}$ trimester pregnancy was detected in 4 cases (5\%), and miscarriage in 2 (3\%). Among the rest, there was 1 hydrosalpinx, 1 vaginal wall injury, 1 vaginal infection, 1 intravaginal foreign body, 1 vaginal abscess, 1 mastitis and 1 breast mass and 3 cases with polycystic ovarian syndrome (Figure 2). Four patients were ad-

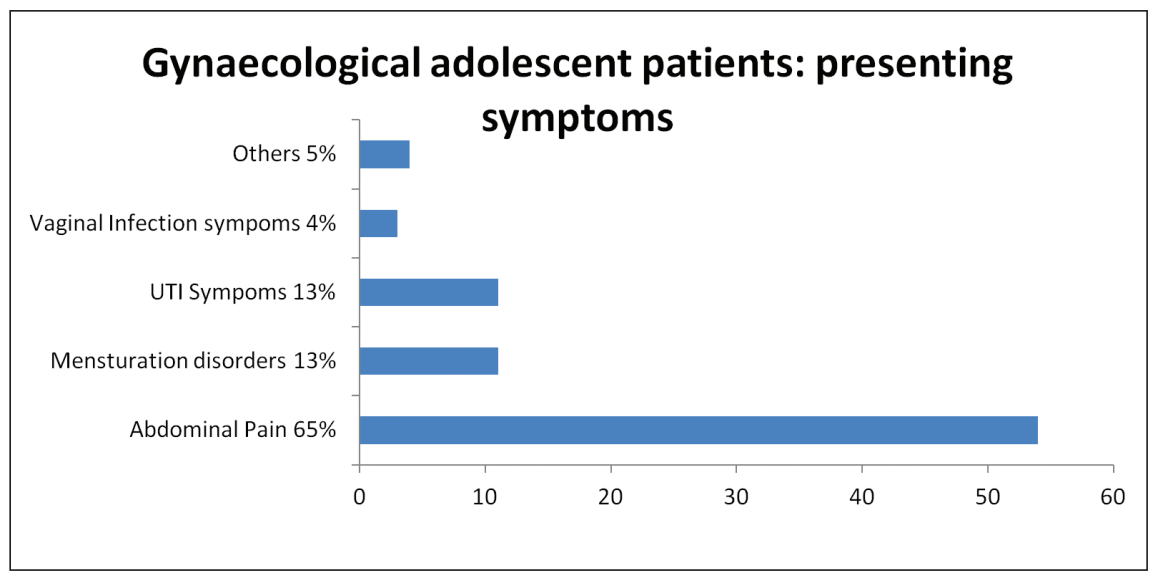

Figure 1. Presenting symptoms of Gynaecological Adolescent Patients in the Gynaecology Emergency Department (ED) of Konstantopouleio General Hospital of Athens (2018). 
mitted in the clinic (5\%), 5 refused admission (6\%), 14 were referred to other specialties $(17 \%)$, and 60 were discharged with instructions (72\%).

Regarding obstetrical cases, the presenting symptom was abdominal pain in 10 adolescents (36\%), hyperemesis in 6 (21\%), myometrial activity in 5 (18\%), vaginal bleeding in $4(14 \%)$, fever in 2 (7\%) and concussion in one (4\%) (Figure 3). In 18 cases $(64 \%)$ no acute medical condition was diagnosed so they were discharged with instructions. Two were referred to other specialties, four were admitted in active labour (14\%) and one with threatened delivery, and three refused the examination (Figure 4).

\section{Discussion}

Adolescents represent $7 \%$ of all the women that visited the O\&G ED during 2018. They constitute a considerable population group and often have spe-

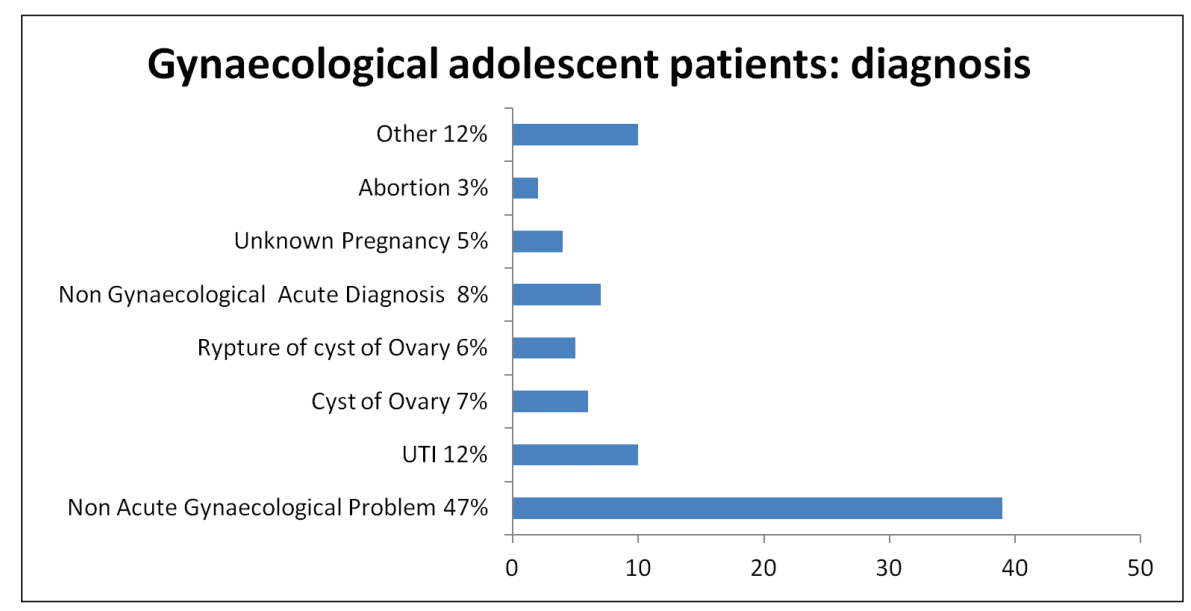

Figure 2. Diagnosis of Gynaecological Adolescent Patients in the Gynaecology Emergency Department of Konstantopouleio General Hospital of Athens (2018).

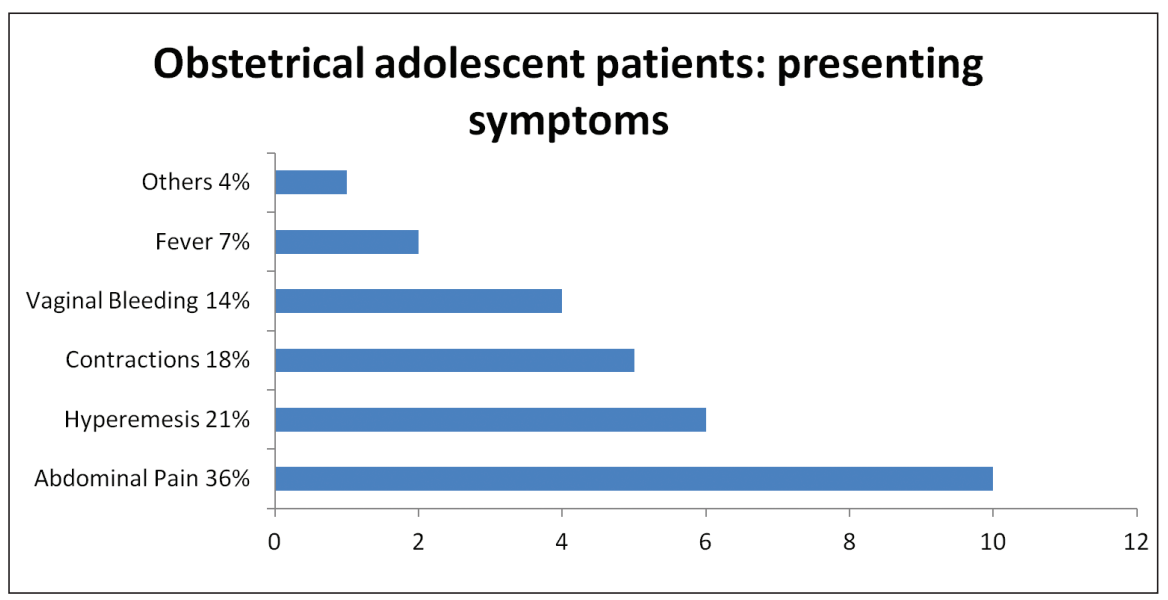

Figure 3. Presenting symptoms of Obstetrical adolescent patients in the Obstetrics Emergency Department of Konstantopouleio General Hospital of Athens (2018). 


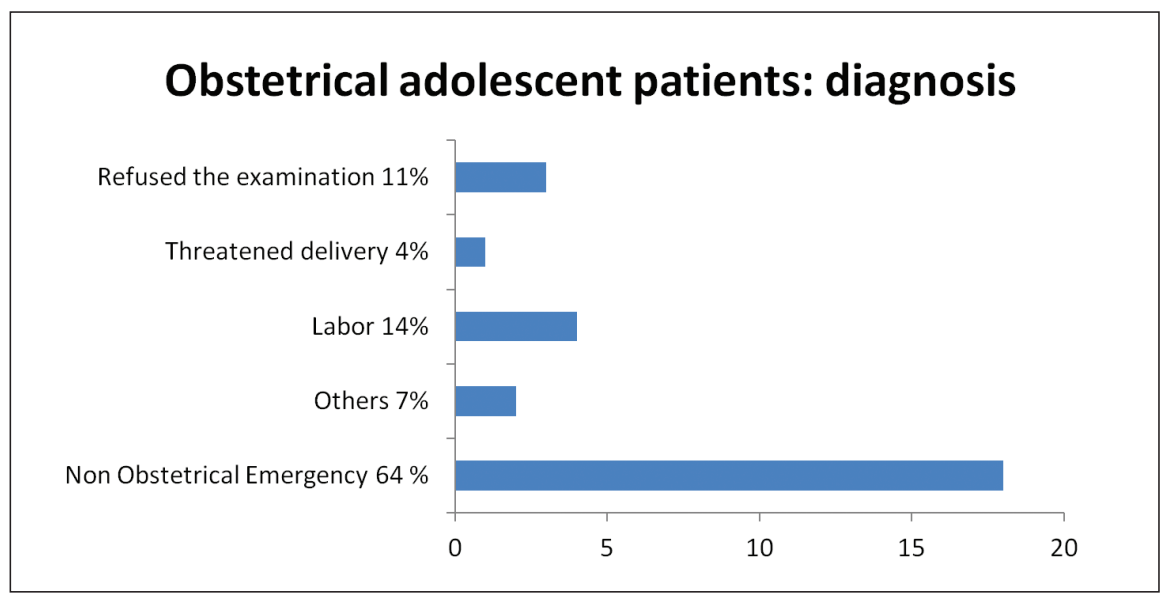

Figure 4. Diagnosis of Obstetrical adolescent patients in the Obstetrics Emergency Department of Konstantopouleio General Hospital of Athens (2018).

cial gynaecological problems that general Obstetricians \& Gynaecologists need to be aware of. Most of the presenting conditions were rather general and could be adequately managed in a general hospital. In nearly half the cases there was no gynaecological pathology diagnosed, which may indicate that young girls and their families are very anxious when gynaecological symptoms are concerned but also underlines the need for better primary healthcare coverage in Greece. Pregnancies are not uncommon in this age group and obstetric conditions need to be managed accordingly.

In our study two thirds of the gynecological cases presented with pelvic pain. Similar results came from a French study ${ }^{5}$ where $55 \%$ of the young women attending O\&G ED complained of pain. Another study from a referral teaching hospital in Athens Greece $^{6}$ identified menstrual disorders as the commonest presenting complain, with pelvic inflammation, ovarian tumors and congenital defects following. This is reasonable, as oligomenorrhea and functional bleeding are common in adolescence due to the immaturity of the YpothalamusYpophysis-Gonad axis, but different to our findings.
Similar results came from an Indian study ${ }^{7,8}$ of 113 adolescents attending $0 \& G$ ED where three fourths complained of menstrual disorders and hardly any of abdominal pain. Other indications were vaginal secretions $(14,3 \%)$, ovarian tumors $(4.5 \%)$, perineal trauma (1.8\%), septic miscarriage $(2.7 \%)$ and sexual harassment (1.8\%). This wide variation in the literature results could be attributed to different populations studied with various educational and social levels and, more likely, to differences between health care systems or even between settings of different hospitals.

Most of the cases in our study, as well as in the literature, had minor $0 \& \mathrm{G}$ problems and some had serious conditions such as pregnancy complications, miscarriage, ovarian cyst, inflammation and trauma. These are similar to what is encountered in the adult population and the current setting could adequately manage them. The Greek National Healthcare System allows direct access to hospitals' emergency departments where the triage is rather elementary. Providing emergency services in the primary setting and upgrading hospitals' emergencies triage could reduce the workload of the specialised departments. 
On the other hand, 0\&G problems by their nature are special and sensitive, even more so when adolescents are concerned, so rapid access can be crucial $^{9}$.

\section{Conclusion}

O\&G emergency conditions in adolescent females are common and potentially serious. Healthcare professionals need to be aware and alert to the special needs of this sensitive population group. Paediatric \& Adolescent Gynaecology is part of the everyday practice and subspecialty services need to be accessible when needed.

\section{References}

1. Who.int.2020.AdolescentHealth.[online]Availableat: https://www.who.int/southeastasia/ health-topics/adolescent-health [Accessed 27 December 2020].

2. Grover SR. Gynaecology problems in puberty. Best Pract Res Clin Endocrinol Metab. 2019 Jun;33(3):101286.

3. Vayngortin T, Kant S. Identification and management of adolescent gynecologic emergencies in the emergency department. Pediatr Emerg Med Pract. 2019 Feb;16(2):1-24.

4. Bedei I, Bumbuliene Z, Sirakov M, Mahmood T, Wood PL; European Association of Paediatric and Adolescent Gynaecology. Provision of paediatric and adolescent gynaecology in Europe today: A joint review by the European Association of Pae- diatric and Adolescent Gynaecology (EURAPAG) and European Board and College of Obstetrics and Gynaecology (EBCOG). Eur J Obstet Gynecol Reprod Biol. 2019 Apr;235:116-120.

5. Gentile, S., Durand, A., Vignally, P., Sambuc, R. and Gerbeaux, P., 2009. Les patients «non urgents » se présentant dans les services d'urgencesont-ilsfavorables à uneréorientationversune structure de soinsalternative ?. Revue d'Épidémiologie et de Santé Publique, 57(1):3-9.

6. Creatsas, G. Adolescent gynecology and obstetrics. European Journal of Obstetrics \& Gynecology and Reproductive Biology, 1995 58(2):107-109

7. Rathod AD, Chavan RP, Pajai SP, Bhagat V, Thool P. Gynecological Problems of Adolescent Girls Attending Outpatient Department at Tertiary Care Center with Evaluation of Cases of Puberty Menorrhagia Requiring Hospitalization. J Obstet Gynaecol India. 2016 Oct;66(Suppl 1):400-6.

8. Pokharel HP, Dahal P, Rai R, Budhathoki S. Surgical emergencies in obstetrics and gynaecology in a tertiary care hospital. JNMA J Nepal Med Assoc. 2013 Jan-Mar;52(189):213-6.

9. Sandy EA 2nd, Kaminski R, Simhan H, Beigi R. Contemporary Obstetric Triage. Obstet Gynecol Surv. 2016 Mar;71(3):165-77.

Received 09-12-20

Revised 17-12-20

Accepted 21-12-20 